\title{
Determination of the Formation of Dark State via Depleted Spontaneous Emission in a Complex Solvated Molecule
}

\author{
Xunmin Guo, Sufan Wang, Andong Xia,* and Hongmei Su* \\ The State Key Laboratory of Molecular Reaction Dynamics, and Beijing National Laboratory for \\ Molecular Sciences (BNLMS), Institute of Chemistry, Chinese Academy of Sciences, Beijing 100080, \\ People's Republic of China
}

Received: January 22, 2007; In Final Form: April 28, 2007

\begin{abstract}
We present a general two-color two-pulse femtosecond pump-dump approach to study the specific population transfer along the reaction coordinate through the higher vibrational energy levels of excited states of a complex solvated molecule via the depleted spontaneous emission. The time-dependent fluorescence depletion provides the correlated dynamical information between the monitored fluorescence state and the SEP "dumped" dark states, and therefore allow us to obtain the dynamics of the formation of the dark states corresponding to the ultrafast photoisomerization processes. The excited-state dynamics of LDS 751 have been investigated as a function of solvent viscosity and solvent polarity, where a cooperative two-step isomerization process is clearly identified within LDS 751 upon excitation.
\end{abstract}

\section{Introduction}

Ultrafast dark processes such as electronic charge redistribution, intramolecular vibrational relaxation (IVR), intermolecular vibrational relaxation, and conformational relaxation dynamics in complex solvated molecules are one of the most common phenomena in physics, chemistry, and biology, which have attracted a great deal of attention, both experimental and theoretical over the past 10 years. ${ }^{1-25}$ These dark processes are commonly treated as fast radiationless phenomena in chemical dynamics, ${ }^{12}$ where a variety of the quantum controls of chemical reactions based on the population of an excited electronic state as a first step with ultrafast femtosecond pulse would strongly suffer from these dark processes. ${ }^{2-7,14,18}$ Although much effort has been devoted with various ultrafast spectral techniques, it is still challenging to experimentalists in determining these dark processes because these dark processes often highly compete with each other, and take place simultaneously on an ultrafast time scale. ${ }^{1-18}$ For instance, a well-known example of the situation where the vibrationally coherent photochemistry in the femtosecond primary event of vision is also involved in a radiationless deactivation process, comes from the investigation of biological photoactive protein Rhodopsin. ${ }^{3,4}$ Similarly, the evolution of some styryl dye molecules from a locally excited (LE) electronic state to a charge transfer state has been studied by numerous authors, ${ }^{12-18}$ who proposed this behavior to one or more of the possible conformational changes which produce the dark sink-like solvation-associated TICT (twisted intramolecular charge transfer) state, but there is no direct observation of the formation of the dark state.

In a series of pioneering control experiments, ${ }^{5,14,18}$ it was shown that with right amount of negative linear chirp, a single excitation pulse comprises a complete pump-dump sequence, where the optimal chirp field is shown to consist of a transform limited blue portion for promoting population to the excited

* Corresponding author. E-mails: andong@iccas.ac.cn, hongmei@ iccas.ac.cn. state, and a negatively chirped red tail for following the evolving population, and dumping it back to ground state through stimulated emission. Although the optimal control experiments are often employed to determine the dynamics on the excited states even without any a priori knowledge on the molecule system, ${ }^{5,14,18,19}$ little was known concerning the involvement of fast solvation and isomerization in determining the population transfer within a superposition of many excited states in the large polyatomic molecules. Typically, the fast solvent motions could always cause irreversible dynamic Stokes shifting of fluorescence, introducing ambiguity into the interpretation of the observed dynamics. Since the intrapulse pump-dump process is sensitive to the laser frequency (energy) and the dynamics of the time-scale of the femtosecond pulse, in many cases, a chirped femtosecond pulse with limited bandwidth in energy concerted the intrapulse pump-dump sequence is somewhat not enough to follow the evolution along the coupling excited electronic states in complex solvated molecules. . $^{514,18}$ Very few works concerned the anisotropic behavior when the decay processes takes place in the region of torsional motion and optically accessed excited states with different dipole transition orientations due to the complex excited-state isomerization.

As a typical laser dye, LDS 751 (styryl 8: 2-(4-(4-dimethylaminophenyl)-1,3-butadienyl)-3-ethylbenzothiazolium perchlorate), is a nonrigid electronic push-pull type molecule. Scheme 1 shows the molecular structure of LDS 751. In polar solvent, the deactivation of the first excited-state is believed to be through the isomerization channel within a few hundred femtoseconds, where a $200 \mathrm{fs}$ isomerization was reported in LDS 750 before, but the mechanism remains unclear to a large extent. ${ }^{17}$ The twisting of LDS 751 in the excited-state may follow a two-step isomerization: the first step is the barrierless torsional motion around the $\mathrm{C}-\mathrm{N}$ bond of dimethyl group, and the second involves a low-barrier isomerization around the central $\mathrm{C}-\mathrm{C}$ bond of butadiene to form a final stabilized sink-like dark TICT state with a complete charge separation. ${ }^{12-18}$ Related to this are 


\section{SCHEME 1: The Molecular Structure of LDS 751}

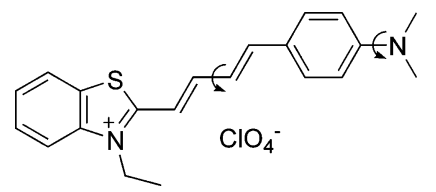

the questions: How to follow the evolution of molecules in excited-state during a multidimensional coordinate with solvation and multitude of intramolecular active modes including torsional motion and isomerization? How to determine the formation of the dark TICT state?

In this paper, we address these questions from a study of LDS 751 as a function of solvent viscosity by employing a powerful pump-dump technique of femtosecond time-resolved stimulated emission pumping fluorescence depletion (FS TR SEP FD). ${ }^{10,11}$ The femtosecond fluorescence depletion anisotropy is further developed to provide complementary information of different dipole transition orientations corresponding to the excited-state isomerization. The time-dependent fluorescence depletion provides the correlated dynamical information between the monitored fluorescence state and the SEP "dumped" dark states, and therefore allow us to obtain the dynamics of the formation of the dark states corresponding to the complex excited-state isomerization.

\section{Materials and Methods}

Materials. The laser dye LDS 751 (styryl 8: 2-(4-(4-dimethylaminophenyl)-1,3-butadienyl)-3-ethylbenzothiazolium perchlorate) is purchased from Lambda Physik GmbH (German). All solvents used are A.R. grade or higher.

Steady-State Spectroscopy. UV/Vis absorption and emission spectra are recorded on a spectrophotometer (UV1601, Shimadzu, Japan) and a fluorescence spectrometer (F4500, Hitachi, Japan), respectively. All the experiments are carried out at ambient temperature.

Preparation of a Series of Polar Aprotic Solvents with Different Viscosities but Constant Polarity. Viscosity studies were done frequently by changing different kind of solvents before, especially for such as glycerol or other alcoholic solvents, which may lead some misleading for analysis of the viscosity effects in chemical reaction because of the complexity (for example, the possible existence of specific hydrogen bond in alcohol solutions) of different solvents. ${ }^{26}$ To avoid this misleading, in this work, we obtain a series of polar aprotic solvents with different viscosities but constant polarity from binary mixture of Acetone and DMSO, where the problem of multiple dielectric relaxation and specific hydrogen-bonding could be avoided. Five different viscosity solvents with constant polarity are prepared for femtosecond fluorescence depletion experiments. In brief, a pair of polar aprotic solvents (acetone and DMSO) with similar polarity ( $\Delta f$ of acetone and DMSO are 0.6485 and 0.655 , respectively) but quite different viscosity $\left(\eta\right.$ of acetone and DMSO are 0.308 and 1.968 , respectively ${ }^{8}$ ) is mixed properly according to different volume ratio. Viscosity values of the mixtures from 0.308 (acetone) to 1.968 (DMSO) were calculated according to eq $1: 27,28$

$$
\ln \eta_{\text {mix }}=\sum_{i=1}^{2} w_{\mathrm{i}} \cdot \ln \eta_{\mathrm{i}}
$$

where $\eta_{\mathrm{i}}$ and $w_{\mathrm{i}}$ represent viscosity and weight component of the individual constituent, respectively). The polarity $(\Delta f)$ of

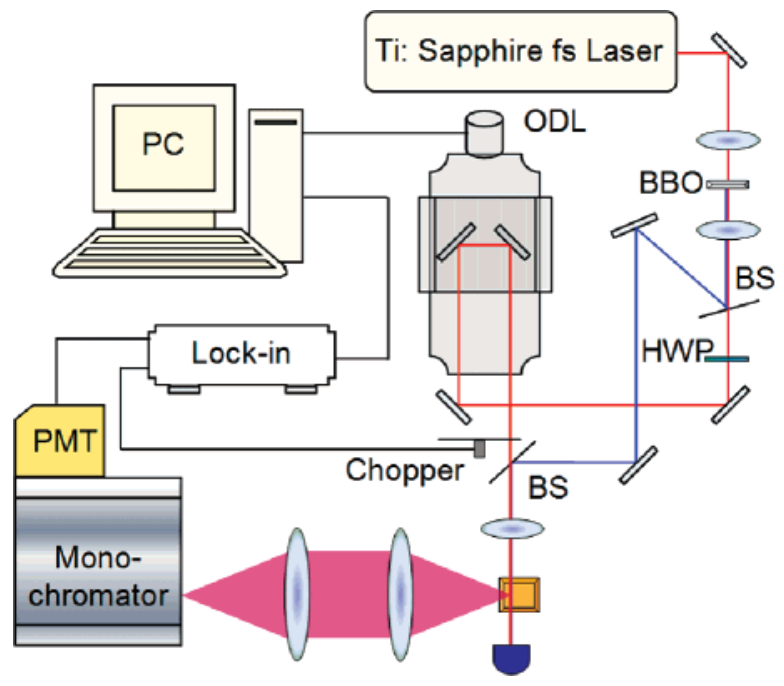

Figure 1. Diagram of the homemade femtosecond time-resolved stimulated emission pumping fluorescence depletion (FS TR SEP FD) setup. HWP, half-wave plate; ODL, optical delayed line; BS, beam splitter.

the DMSO/acetone mixture was calculated according to eq $2: 29-31$

$$
\Delta f=\frac{(\epsilon-1)}{(2 \epsilon+1)}-\frac{\left(n^{2}-1\right)}{\left(2 n^{2}+1\right)}
$$

where $\Delta f$ is the orientational polarizability of solvent which could deduced from the dielectric constant $(\epsilon)$ and the refractive index $(n)$ of the solvent represented by eq 2 . The corresponding $\epsilon_{\text {mix }}$ and $n_{\text {mix }}$ for the mixed solvent of DMSO/acetone are then calculated according to $\epsilon_{\text {mix }}=f_{\mathrm{a}} \epsilon_{\mathrm{a}}+f_{\mathrm{b}} \epsilon_{\mathrm{b}}$ and $n_{\text {mix }}{ }^{2}=f_{\mathrm{a}} n_{\mathrm{a}}{ }^{2}+$ $f_{\mathrm{b}} n_{\mathrm{b}}{ }^{2}$, respectively. ${ }^{32}$ The concentration of LDS 751 in different solvents is about $0.16 \mathrm{mM}$ for all the ultrafast experiments.

Femtosecond Fluorescence Depletion Measurements. Fluorescence depletion from LDS 751 is measured on a homemade femtosecond time-resolved stimulated emission pumping fluorescence depletion (FS TR SEP FD) setup based on the principle of so-called "pump-probe" technology described elsewhere. ${ }^{10}$ Figure 1 shows the diagram of the homemade FS TR SEP FD setup. In brief, A CW laser with $532 \mathrm{~nm}$ (Verdi-V5, Coherent, USA) pumps a Ti:sapphire laser (Mira 900S, Coherent, USA) producing the pulse at $800 \mathrm{~nm}$ with duration of $50 \mathrm{fs}$ at a repetition of $76 \mathrm{MHz}$. The output power is about $650 \mathrm{~mW}$. This fundamental beam was led into a $0.5 \mathrm{~mm}$ thick BBO crystal to produce a second harmonic beam at $400 \mathrm{~nm}$. The second harmonic beam and the residual fundamental beam are split by a dichromic mirror. The ultrafast double-frequency beam at 400 $\mathrm{nm}\left(84.5 \mathrm{MW} / \mathrm{cm}^{2}\right)$ is used for pumping the sample solution to generate the Franck-Condon state, then, the residual ultrafast fundamental pulses at $800 \mathrm{~nm}\left(1.69 \mathrm{GW} / \mathrm{cm}^{2}\right)$ with specific polarization (modulated by rotating a zero-level half-wave plate) are introduced as a probe beam to "dump" the population of dye molecules from excited states after a variable delay time. The spontaneous fluorescence emission is perturbed due to compulsive stimulated emission depletion (STED); i.e., the fluorescence is depleted in the presence of "dumping" pulse. The relative fluorescence intensity depletion at the monitored fluorescence wavelength as a response of delay time is detected via a PMT coupled monochromator. A lock-in amplifier is employed to extract the depletion signal correlated to the "dumping" beam, where the relative amounts of the fluorescence quenching are known to be proportional to the amplitudes of 


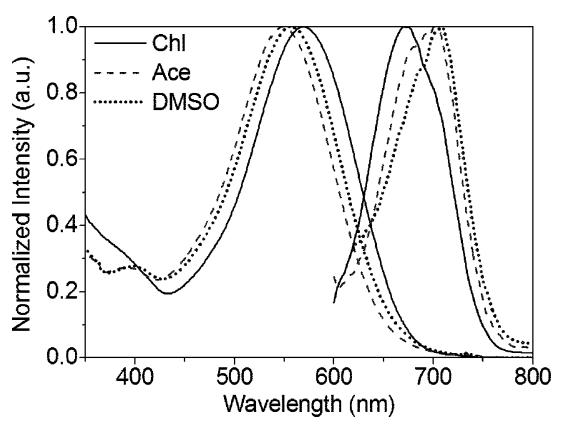

Figure 2. Steady-state absorption and emission spectra of LDS 751 in chloroform, acetone, and DMSO. The excitation wavelength of fluorescence spectra is $520 \mathrm{~nm}$.

the fluorescence spectrum at the quenching wavelength. ${ }^{33}$ Therefore, the change in fluorescence intensity observed should be the difference in the integrated fluorescence intensity at monitored emission wavelength before and after SEP. To avoid the possible thermal effects and photobleaching upon intense femtosecond pulse, sample solution is fluctuated with a highspeed rotor in the sample cell to increase the $\mathrm{S} / \mathrm{N}$ ratio of detection. In pervious depletion measurement by Prof. Kong's group in our laboratory, ${ }^{10,11,34}$ they only employed parallel polarized beam as pumping beam and vertical polarized probe beam as dumping beam, where the crossed polarization SEP was less efficient when the dipole transition of the molecule was changed after an initial short pulse excitation. In order to get full information about the ultrafast dynamics, we here develop the isotropic $\left(54.7^{\circ}\right.$ ) and anisotropic (parallel and vertical) femtosecond fluorescence depletion methods, where the polarizations of dumping beams with respect to that of pumping beam are selected by rotating a zero-level half-wave plate to obtain isotropic $\left(54.7^{\circ}\right)$ and anisotropic (parallel and vertical) results, respectively. The time-resolved anisotropic decay $r(t)$ was calculated from the decay curves under the condition of parallel polarizations $\left(I_{||}\right)$and perpendicular polarizations $\left(I_{\perp}\right)$ of dumping beam relative to the polarization of pumping beam according to eq $3:^{33,35,36}$

$$
r(t)=\frac{I_{\|}(t)-G \cdot I_{\perp}(t)}{I_{\|}(t)+2 G \cdot I_{\perp}(t)}
$$

The factor $G$ accounts for the difference in sensitivities for the detection of signal in the perpendicular- and parallelpolarized configurations, where $G\left(G=I_{\perp} / I_{\|}\right.$, when the excitation is vertically polarized) is estimated about 1.02 . The instrument response function is determined according to the 1 $+1^{\prime}$ two photon excited fluorescence method as described before, ${ }^{10,11,34}$ in which, the IRF (instrumental response function) up to $90 \mathrm{fs}$ is achieved. All the temporal evolution profiles are fitted by the convolution between the IRF with a multiexponential function according to iterative deconvolution by FluoFit software based on the Levenberg-Marquardt and Simplex algorithms (Version 3.3, PicoQuant, Germany). The fitting quality is judged by weighted residuals and reduced $\chi^{2}$ values.

\section{Results and Discussion}

Steady-State Spectra. Figure 2 shows the steady state absorption and emission spectra of LDS 751 in acetone and DMSO. For comparison, the absorption and emission spectra of LDS 751 in weak polar aprotic chloroform are also shown in Figure 2. The absorption spectra of LDS 751 in all solutions are structureless and broad, whereas the fluorescence spectra show the dual-peak feature around 680 and $730 \mathrm{~nm}$, indicating

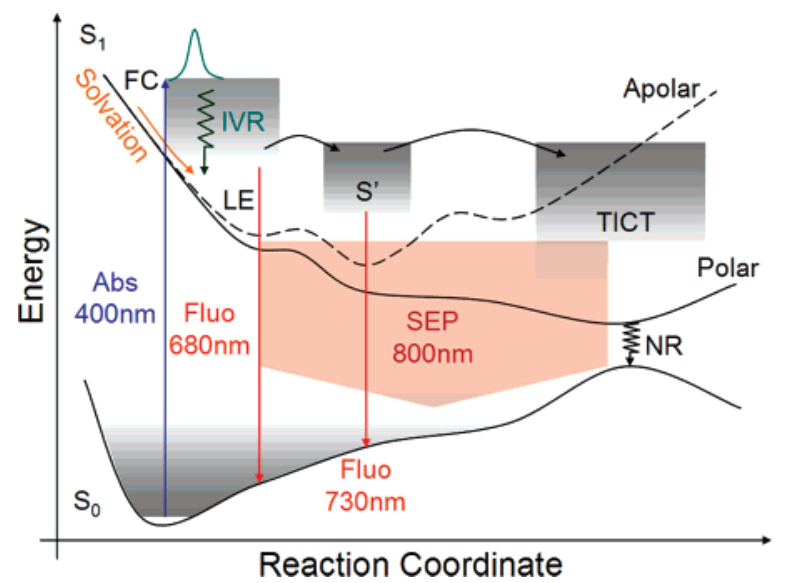

Figure 3. A schematic representation of the potential-energy curves for the relaxation of LDS 751 in the $S_{1}$ state. The dash curve and the solid curve represent the potential energy surfaces of the $S_{1}$ state in weak polar aprotic chloroform and polar aprotic DMSO/acetone, respectively. The broad shaded arrow area shows that the efficient SEP occurs after the excited-state wave packet moves to the upper of monitored fluorescence state with the energy in resonance with the "dumping" beam in the process of formation of the dark TICT state.

the involvement of charge transfer and/or isomerization occurred in LDS 751. Compared to the absorption and emission spectra of LDS 751 in weak polar aprotic chloroform, the maxima of absorption shift to the blue and those of fluorescence shift to the red were observed when LDS 751 was dissolved in polar aprotic acetone and DMSO solvents, indicating the decreased solvent stabilization of $S_{1}$ relative to $S_{0}$ and increased charge separation in the excited state. ${ }^{37}$ The large Stokes shift has been reported as the result of solvation and conformational relaxation correlated to the solvent polarity. To tentatively assign the dual fluorescence peaks around 680 and $730 \mathrm{~nm}$, the potential energy surface calculations along $\mathrm{C}-\mathrm{C}$ and $\mathrm{C}-\mathrm{N}$ twisting coordinates of LDS 751 have been carried out for the ground state and excited states by using density functional theory (DFT) and timedependent DFT (TD-DFT) methods as implemented in the Gaussian 03 package. ${ }^{38}$ Detailed calculation results on LDS 751 are shown in Supporting Information. From the calculated potential energy surfaces of the LUMO and HOMO states of LDS 751 in gas phase with different torsion degrees around central $\mathrm{C}-\mathrm{C}$ single bond of butadiene or $\mathrm{C}-\mathrm{N}$ bond of end group, respectively, it is found that direct isomerization along the central $\mathrm{C}-\mathrm{C}$ bond (see Figure S3 in Supporting Information, step from $b$ to $c$ ) seems a little difficult since the twisting barrier is a little higher that that of the twisting of $\mathrm{C}-\mathrm{N}$ bond of end group. Although our calculations have not taken the solvent effects into account, at least from the qualitative point of view, it is clear that a cooperative two-step isomerization of LDS 751 could be encouraged as predicted by molecular orbital calculations, where the first step is nearly barrierless torsional motion around the $\mathrm{C}-\mathrm{N}$ bond of dimethyl group, and the second involves a low-barrier isomerization around the central $\mathrm{C}-\mathrm{C}$ bond of butadiene to form a final stabilized sink-like dark TICT state with a complete charge separation (See Figure S2 and Figure S3 in Supporting Information). Similar two-step isomerization has also be reported in other styryl dyes such as LDS $750^{17}$ and DCM, ${ }^{39-41}$ as well as other push-pull molecule such as DCS. ${ }^{42}$

Figure 3 shows the proposed potential energy curves of LDS 751 in both weak polar and polar solvents. The dual fluorescence peaks around 680 and $730 \mathrm{~nm}$ of LDS 751 are from LE state and intermediate ICT state $\left(S^{\prime}\right)$, respectively (see Supporting Information). Since the deactivation of the first excited-state of 
LDS 751 in polar solvent is believed to be through the isomerization channel within a few hundred femtoseconds as reported in a similar dye $\operatorname{LDS} 750,{ }^{17}$ which is faster than typical vibrational dephasing and relaxation times, ${ }^{3,4}$ the observed dual fluorescence at 680 and $730 \mathrm{~nm}$ are expected to occur from nonstationary, vibrationally unrelaxed excited-state populations. To determine these properties, femtosecond fluorescence depletion measurements further provide the solvent-dependent dynamics of the formation of the dark states corresponding to the cooperative two-step photoisomerization processes in the excited states.

Femtosecond Fluorescence Depletion Dynamics of LDS 751. Following a $400 \mathrm{~nm}\left(84.5 \mathrm{MW} / \mathrm{cm}^{2}\right)$ femtosecond pulse excitation of the molecule, a delayed near-infrared (NIR) laser beam at $800 \mathrm{~nm}\left(1.69 \mathrm{GW} / \mathrm{cm}^{2}\right)$ far from the monitored fluorescent state is introduced selectively to follow the evolving population, and "dump" the reactive excited-state back to the ground state, where only SE is conducted without simultaneous excited-state absorption. ${ }^{17}$ Since the isomerization is so fast within a few hundred femtoseconds, ${ }^{17}$ which is faster than typical vibrational dephasing and relaxation times, the population transfer along the reaction coordinate through the higher vibrational energy levels among different excited states must be considered during isomerization because of the large excess photon energy. The ultrafast deactivation through selective SEP processes could occur from the vibrationally unrelaxed excitedstate populations. ${ }^{3,4}$ Generally, SEP can occur immediately following an initial Franck-Condon excitation. In fact, from the principle of the data construction of FS TR SEP FD (see Supporting Information), the stimulated emission (SE) cannot be observed before the formation of the monitored fluorescence states at 680 or $730 \mathrm{~nm}$ because depletion signal is only contributed from the change in fluorescence intensity of the monitored fluorescence states before and after SEP. The timedependent depletion of spontaneous emission at the monitored fluorescent state provides the information of the population propagated on the excited states, which offers the advantage to selectively extract the correlated dynamical information between the monitored fluorescence state and the SEP "dumped" reactive excited states. The polarization and frequency (energy) of the dump beam for stimulated emission pumping can be selected to focus mainly on the specific excited states together with the circumvention of electric dipole selection rules corresponding to the information on molecular structure, dynamics and geometry, where the isomerization could lead to the dipole transition changes of excited states. In the case of LDS 751 with the dump beam at $800 \mathrm{~nm}$, only final dark state was mainly dumped through stimulated emission. ${ }^{17}$ This selectivity aids in the understanding of the very early dynamics of the excitedstate evolution, including the ultrafast excited-state isomerization. $^{5,12-18}$

Figure 4 a shows the typical fluorescence depletion of LDS 751 monitored at $730 \mathrm{~nm}$. The isotropic depletion decays are well fitted with a double-exponential function. Table 1 lists all the fitted results of LDS 751 in acetone, DMSO and in the binary mixture of DMSO/acetone with different viscosities. The initial ultrafast decay $\left(\tau_{1}\right)$ less than $1 \mathrm{ps}$ is attributed to vibrational relaxation processes in the monitored fluorescence state, ${ }^{10,11}$ whereas the slow decay $\left(\tau_{2}\right)$ around $1-2$ ps is assigned to solvation-assisted charge transfer and conformational relaxation, which is strongly viscosity-dependent. Furthermore, no significant difference in the dynamics at both 680 and $730 \mathrm{~nm}$ are observed (see Figure S4 in Supporting Information), indicating the very fast barrierless crossing process from $\mathrm{FC} /$

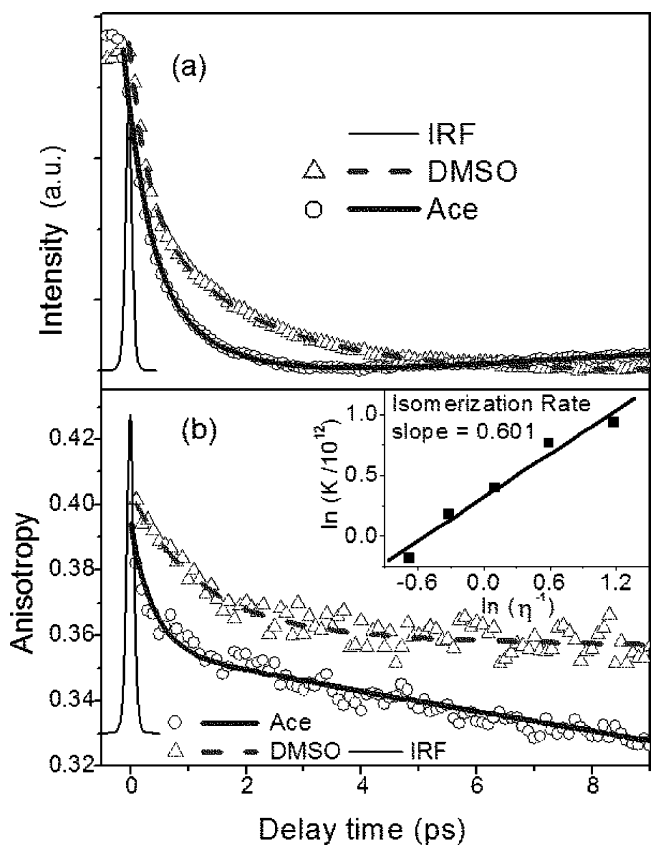

Figure 4. Femtosecond isotropic (a) and anisotropic (b) fluorescence depletion of LDS 751 monitored at $730 \mathrm{~nm}$. Inset of panel b shows the viscosity $(\eta)$ dependence of isomerization rate $(k)$. The dots and lines represent the experimental and fitted results, respectively. The instrument response function (IRF) is also shown at time zero.

TABLE 1: Fitted Results of the Isotropic and Anisotropic Decays of LDS 751 in Solvents with Various Polarities $(\Delta f)$ and Viscosities $(\eta)$

\begin{tabular}{ccccc}
\hline solvent $(\Delta f)$ & $\eta(\mathrm{cP})$ & $\tau_{1}(\mathrm{fs})$ & $\tau_{2}(\mathrm{ps})$ & $\tau_{\text {ani }}(\mathrm{ps})$ \\
\hline DMSO/acetone $(0.66)$ & 1.968 & $203(65 \%)$ & $1.93(35 \%)$ & 1.19 \\
& 1.374 & $206(63 \%)$ & $1.65(37 \%)$ & 0.83 \\
& 0.905 & $198(60 \%)$ & $1.41(40 \%)$ & 0.67 \\
& 0.554 & $207(54 \%)$ & $1.07(46 \%)$ & 0.46 \\
chloroform (0.29) & 0.308 & $201(50 \%)$ & $0.92(50 \%)$ & 0.39 \\
& 0.539 & $222(80 \%)$ & $4.28(20 \%)$ & 0.27
\end{tabular}

LE to the intermediate isomeric $\mathrm{S}^{\prime}$ state by twisting the $\mathrm{C}-\mathrm{N}$ bond of end group of LDS 751 through the higher vibrational energy levels of excited states.

To further explore the solvation and conformational relaxation dynamics, we perform the anisotropic depletion measurements. As a nonrigid molecule of LDS 751, the solvation is coupled to the bond twisting, leading to a large change of the dipole moment. The anisotropic depletion provides the information on not only the formation of final dark TICT state correlated with photoisomerization but also solvation dynamics. Figure. $4 \mathrm{~b}$ shows the fluorescence anisotropic depletion of LDS 751. The anisotropic depletion decay of LDS 751 in both acetone and DMSO is fitted satisfactorily with a single-exponential function (see Table 1). It is found that the anisotropic decay of LDS 751 in lower viscosity acetone is fast with a time constant $\left(\tau_{\text {ani }}\right)$ only about $0.39 \mathrm{ps}$, whereas, that in higher viscosity DMSO is slow about 1.19 ps. The high solvent viscosity does, indeed, slow down the isomerization process.

Since both acetone and DMSO have almost the same polarities, thus following a pulse excitation, the LDS 751 molecule is expected to reach the similar minima of relaxed excited-state but with different time constants during solvation. The observed anisotropy results from the collective contributions from the solute-contained solvent-cage, where the coupled twisting around both the $\mathrm{C}-\mathrm{N}$ bond of the end-group and the central $\mathrm{C}-\mathrm{C}$ bond produces a complete charge transfer state with a large dipole moment. A final relaxed dark TICT state is 


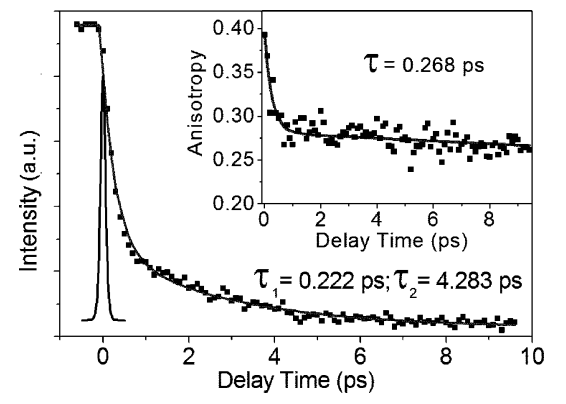

Figure 5. Isotropic and anisotropic (inset) fluorescence depletion signals of LDS 751 in weak polar chloroform monitored at $730 \mathrm{~nm}$. The IRF is also shown at time zero.

then reached, which is stabilized by the polar solvent. Compared to the solute in DMSO, the solvation is much faster in lower viscous acetone, leading to the faster decrease of the total available energy in the molecule above the central $\mathrm{C}-\mathrm{C}$ twisting barrier. The observed fast dynamics of the anisotropic decays with time constants about $0.39 \mathrm{ps}$ in acetone and $1.19 \mathrm{ps}$ in DMSO appear to be the "signature" of a low-energy barrier state essentially undergoing population transfer through the higher vibrational energy levels of excited states with two coupled torsional motions around both the $\mathrm{C}-\mathrm{N}$ bond of the end-group and the central $\mathrm{C}-\mathrm{C}$ bond, which are responsible for the processes of the formation of the relaxed dark TICT states. Meanwhile, the inset of Figure 4b shows the viscositydependent anisotropic rates $(k)$ corresponding to the isomerization processes, which was fitted according to the KramersSmoluchowski equation, ${ }^{43,44} k \propto 1 / \eta^{\alpha}$, where $\eta$ is the solvent viscosity. The value of $\alpha$ obtained, about 0.60 , by the fitting further indicates such a low-energy barrier during isomerization. ${ }^{37}$

The barrier-crossing flux could be promoted by the faster solvation and SEP. The stimulated emission then become efficient when the wave packets move to the final relaxed dark TICT state surface after the faster decrease of the total available energy in the molecule above the central $\mathrm{C}-\mathrm{C}$ twisting barrier, leading to the faster isomerization about $0.39 \mathrm{ps}$ in low viscous acetone compared to the slower isomerization about $1.19 \mathrm{ps}$ in high viscous DMSO. This is in agreement with the isotropic results where the solvation-assisted conformational relaxation has a large amplitude $(50 \%) \mathrm{C}-\mathrm{C}$ twisting with time constant about $0.92 \mathrm{ps}$ in acetone and a small amplitude (35\%) $\mathrm{C}-\mathrm{C}$ twisting with time constant about $1.93 \mathrm{ps}$ in DMSO as shown in Table 1.

To further determine how the solvation influences the twisting of solute, a weak polar aprotic chloroform solvent (much less polar compared to DMSO/acetone) is chosen for comparison. With the isotropic and anisotropic depletion measurements as shown in Figure 5, a slow 4.28 ps solvation and a fast 0.27 ps vibrational relaxation process are clearly identified for LDS 751 at the monitored fluorescence state in chloroform. The slow process about $4.28 \mathrm{ps}$ is a typical solvation time for chloroform, which is in agreement with the solvation time about $3.70 \mathrm{ps}$ for coumarin 153 in chloroform. ${ }^{8}$ The observed isotropic decay component about 222 fs with large amplitude up to $80 \%$ mainly results from the vibrational relaxation of the intermediate charge transfer state $\left(\mathrm{S}^{\prime}\right)$. Importantly, the conformational relaxation time constant about 0.27 ps for the anisotropic depletion is identical to the time constant about 222 fs for the fast component of the isotropic depletion, indicating that the depolarization of the LDS 751 is mainly from the rotation about the $\mathrm{C}-\mathrm{N}$ of the end-group. Compared to the slow anisotropic decay of LDS 751 with two-step isomerization around both the $\mathrm{C}-\mathrm{N}$ bond of the dimethyl group and the central $\mathrm{C}-\mathrm{C}$ bond in more polar DMSO/ acetone solvents, the fast anisotropic decay of LDS 751 in weak polar chloroform may be explained by a fast barrierless torsional motion only around the $\mathrm{C}-\mathrm{N}$ bond of dimethyl group because of the weak coupling between solute and solvent. Furthermore, in the weak or apolar aprotic solvent, the total available energy above the $\mathrm{C}-\mathrm{C}$ twisting barrier moves up because of the lack of stabilization by the solvent. It is difficult to form the final relaxed dark TICT state through the twisting of the central $\mathrm{C}-\mathrm{C}$ bond, therefore, the conformational relaxation mainly terminates in the intermediate $S^{\prime}$ state in chloroform.

\section{Conclusions}

In conclusion, we report the powerful pump-dump methods to investigate the specific ultrafast population transfer within a superposition of excited states of a complex solvated molecule, where anisotropic control is developed to follow the torsional motion and optically accessed excited states with different dipole transition orientations during a cooperative two-step isomerization process. The time-dependent depletion of the spontaneous emission provides the correlated dynamical information between the monitored fluorescence state and the SEP "dumped" reactive exited state, and therefore offers the advantage of mapping the energy flow from an initial excited-state to one or more other coupled excited electronic states in the molecule. Finally, we would like to stress one point that, the final relaxed TICT state is a sink-like dark state which cannot be observed by normal fluorescence methods because of the nature of radiationless transition. The stimulated emission (SE) lifetime is much shorter even than that of the radiationless TICT state, where SEP process is completed within the laser pulse durations. Therefore, the SEP could be a suitable "probe" to determine the dynamics of the formation of the relaxed dark TICT state. The time-dependent depletion at the monitored fluorescent excited-state provides the information of the population propagated from the monitored fluorescence states toward the final dark TICT state. It could become a general approach to determine the complicated spectral characteristics in various complex solvated molecules with this powerful technique, and thus allow us to determine the dynamics of the formation of the final dark TICT state. For example, this method could also be extended alternatively to study the cis-trans isomerization of the retinal protonated Shiff base (PSB) chromophore embedded in rhodopsin, ${ }^{3,4,45,46}$ where the ultrafast reactions occur from the vibrationally unrelaxed excited-state population. In this case, "SEP" could also be the powerful "probe" in determining the ultrafast photochemical process corresponding to the excitedstate torsional motion.

Acknowledgment. We thank Prof. Fan'ao Kong for his constant encouragement and for many valuable discussions. We also thank referees for their valuable suggestions for revising the manuscript. This work was financially supported by NSFC (No.90306013, No.20473100 and No.20673126), Chinese Academy of Sciences (Y2005018), and National Basic Research Program of China (2006CB806000).

Supporting Information Available: Experimental details including quantum chemical calculation and additional observations. This material is available free of charge via the Internet at http://pubs.acs.org.

\section{References and Notes}

(1) Pal, S. K.; Zewail, A. H. Chem. Rev. 2004, 104, 2099. 
(2) Nibbering, E. T. J.; Wiersma, D. A.; Duppen, K. Phys. Rev. Lett. 1992, 68, 514.

(3) Schoenlein, R. W.; Peteanu, L. A.; Mathies, R. A.; Shank, C. V. Science 1991, 254, 412.

(4) Wang, Q.; Schoenlein, R. W.; Peteanu, L. A.; Mathies, R. A.; Shank, C. V. Science 1994, 266, 422 .

(5) Cerullo, G.; Bardeen, C. J.; Wang, Q.; Shank, C. V. Chem. Phys. Lett. 1996, 262, 362.

(6) Sukharev, M.; Seideman, T. Phys. Rev. Lett. 2004, 93, 093004.

(7) Montagna, M.; Pilla, O.; Viliani, G. Phys. Rev. Lett. 1980, 45, 1008

(8) Horng, M. L.; Gardecki, J. A.; Papazyan, A.; Maroncelli, M. J. Phys. Chem. 1995, 99, 17311.

(9) Kobayashi, T.; Saito, T.; Ohtani, H. Nature (London) 2001, 414, 531.

(10) Zhong, Q. H.; Wang, Z. H.; Sun, Y.; Zhu, Q. H.; Kong, F. A. Chem. Phys. Lett. 1996, 248, 277.

(11) He, Y.; Xiong, Y. J.; Wang, Z. H.; Zhu, Q. H.; Kong, F. A. J. Phys. Chem. A 1998, 102, 4266.

(12) Glasbeek, M.; Zhang, H. Chem. Rev. 2004, 104, 1929 and references therein.

(13) Palit, D. K.; Singh, A. K.; Bhasikuttan, A. C.; Mittal, J. P. J. Phys. Chem. A 2001, 105, 6294.

(14) Bardeen, C. J.; Wang, Q.; Shank, C. V. Phys. Rev. Lett. 1995, 75, 3410.

(15) Hebert, P.; Baldacchino, G.; Gustavsson, T.; Mialocq, J. C. Chem. Phys. Lett. 1993, 213, 345.

(16) Blanchard, G. J. J. Chem. Phys. 1991, 95, 6317.

(17) Kovalenko, S. A.; Ernsting, N. P.; Ruthmann, J. J. Chem. Phys. 1997, 106, 3504.

(18) Nahmias, O.; Bismuth, O.; Shoshana, O.; Ruhman, S. J. Phys. Chem. A 2005, 109, 8246.

(19) Teranishi, Y.; Phys. Rev. Lett. 2006, 97, 053001.

(20) Vengris, M.; vanStokkum, I. H. M.; He, X.; Bell, A. F.; Tonge, P. J.; vanGrondelle, R.; Larsen, D. S. J. Phys. Chem. A 2004, 108, 4587.

(21) Kovalenko, S. A.; Ruthmann, J.; Ernsting, N. P. J. Chem. Phys. 1998, 109, 1894.

(22) Ruhmann, S.; Hou, B. X.; Friedman, N.; Ottolenghi, M.; Sheves,

M. J. Am. Chem. Soc. 2002, 124, 8854.

(23) Ingemann, D.; Ernsting, N. P. J. Chem. Phys. 1995, 102, 2691.

(24) Mondal, J. A.; Ghosh, H. N.; Ghanty, T. K.; Mukherjee, G. T.; Palit, D. K. J. Phys. Chem. A 2006, 110, 3432.
(25) Maciejewski, A.; Naskrecki, R.; Lorenc, M.; Ziolek, M.; Karolczak, J.; Kubicki, J.; Matysiak, M.; Szymanski, M. J Mol. Struct. 2000, 555, 1. (26) Nikowa, L.; Schwarzer, D.; Troe, J. J. Chem. Phys. 1992, 97, 4827. (27) Perry, R. H.; Green, D. W.; Maloney, J. O. In Perry's Chemical Engineer's Handbook; McGraw- Hill: New York, 1984.

(28) Haidekker, M. A.; Brady, T. P.; Lichlyter, D.; Theodorakis, E. A. Bioorg. Chem. 2005, 33, 415.

(29) Lippert, V. E. Z. Elektrochem. 1957, 61, 962

(30) Mataga, N.; Kaifu, Y.; Koizumi, M. Bull. Chem. Soc. Jpn. 1956, $29,465$.

(31) Mataga, N. Bull. Chem. Soc. Jpn. 1963, 36, 654.

(32) Singh, M. K.; Pal, H.; Bhasikuttan, A. C.; Sapre, A. V. Photochem. Photobiol. 1998, 68, 32.

(33) Gryczynski, I.; Kusba, J.; Gryczynski, Z.; Malak, H.; Lakowicz, J. R. J. Phys. Chem. 1996, 100, 10135 .

(34) Zhong, Q. H.; Wang, Z. H.; Liu, Y. Q.; Zhu, Q. H.; Kong, F. A. J. Chem. Phys. 1996, 105, 5377.

(35) Lakowicz, J. R.; Gryczybki, I.; Bogdanov, V.; Kdba, J. J. Phys. Chem. 1994, 98, 334.

(36) Lakowicz, J. R. In Principle of Fluorescence Spectroscopy; Plenum: New York, 1999.

(37) Kamlet, M. J.; Abboud, J. L. M.; Taft, R. W. In Progress in Physical Organic Chemistry; Taft, R. W., Ed.; Interscience: New York, 1981; Vol. $13, \mathrm{p} 485$.

(38) Frisch, M. J. Gaussian 03, Revision B.05; Gaussian, Inc.: Pittsburgh, PA, 2003.

(39) VanTassle, A. J.; Prantil, M. A.; Fleming, G. R. J. Phys. Chem. B, in press.

(40) Marguet, S.; Mialocq, J. C.; Millie, P.; Berthier, G.; Momicchioli, F. Chem. Phys. 1992, 160, 265.

(41) Chang, C. W.; Kao, Y. T.; Diau, E. W. G. Chem. Phys. Lett. 2003, 374,110 .

(42) Abraham, E.; Oberle, J.; Jonusauskas. G.; Lapouyade, R.; Rulliere, C. Chem. Phys. 1997, 214, 409.

(43) Kramers, H. A. Physica 1940, 1, 284

(44) Chandraselhar, S. Rev. Mod. Phys. 1943, 15, 1.

(45) Kochendoerfer, G. G.; Mathies, R. A. J. Phys. Chem. 1996, 100, 14526

(46) Prokhorenko, V. I.; Nagy, A. M.; Waschuk, S. A.; Brown, L. S.; Birge, R. R.; Miller, R. J. D. Science 2006, 313, 1257. 